\title{
The Susceptible Size of Coronavirus Spread, Epidemic Age, and Optimal Decisions
}

\author{
Abdulaziz Omar A. Jughaiman, \\ King Saud University, Saudi Arabia
}

Doi:10.19044/esj.2020.v16n21p150 URL:http://dx.doi.org/10.19044/esj.2020.v16n21p150

\begin{abstract}
This paper aims to determine the susceptible size used in the mathematical model that predicts the epidemic curve. It also aims to ascertain the age of the epidemic and its reliability system, further suggesting optimal decisions. This paper employs the mathematical induction and deduction methods to extract the parameter $\lambda$ and the age of the epidemic. Functions techniques were also used to evaluate the susceptible size. It used the epidemiological forecasting models with $\mathrm{r}$ at 0.15 and $\leq 150$ for Hubei, and $\leq 83$ for Germany. It also used the shortest path and available data to calculate the recovery rate used in mixed strategies game with the decisions trees. This paper found that the susceptible size that added to infection size, to give the $\mathrm{N}$ size, should be determined by a function. This paper found that the longer the epidemic age, the greater the size that affects the virus reliability system. The virus spread active center is a community of unlimited and variable random. This means that is small and almost constant. The optimal decisions are to use the second strategy until the first strategy is available. The second strategy has two parts: searching for vaccine and accurate medicine and applying supportive therapy and protection. This paper therefore suggests explanations to many concepts that will contribute to a better understanding of many phenomena in modeling, epidemic, decisions, economic, and politics.
\end{abstract}

Keywords: Mathematical Models, Epidemic, Coronavirus, Economic, Politics

\section{Introduction}

Mathematical models of the epidemics depend on the variables entered. The most important is the size of the population. The size of the population, which consists of the infected and susceptible, depends on several factors and the most prominent are the type of the epidemic and its history (a modern or an old). Therefore, this paper aims to determine the size of the population, and specifically the size of people who are susceptible to infection 
more accurately so that it is close to reality. Diseases differ greatly in the speed with which they spread. Respiratory diseases (flu, the common cold) are often spread by droplet infection, and a strong uncovered sneeze in a crowd could infect quite a few people. By contrast, diseases that spread by body contact, such as venereal diseases, generally spread more slowly as an epidemic. This spreads from persons with the disease (infectives) to persons who do not yet have it (susceptible). The mathematical theory of epidemics attempts to predict the cause of an epidemic, including such factors as the total number who will be infected and the time when the epidemic will peak. Scientific predictions or estimates often have a misleading impression of certainty. The reason is because manageable models are very often deterministic, i.e., they leave out chance factors. The world is characterized as a place that cannot be known with certainty. The world is a rather uncertain place. Scientists disagree about why this is so. One point of view is called determinism. The alternative view is that the laws of nature have a built-in uncertainty. Whether or not nature is inherently uncertain, it certainly appears uncertain (Meyer Walter, 1984). However, the determinist viewpoint is supposed to be particularly valid. Also, belief in determinism does not reject the experiment. In addition, the experiment may not be able to insert all its elements while the opinion related to experience is supposed to be generally valid. Einstein stated that God does not play dice. For example, the experiment of throwing a coin several times gives the same result if it is thrown in the same conditions with the most accurate details, including the precise surface lines that will be thrown over it and the moment at which it was thrown. Therefore, the use of probability theory is a means of a description, not an individual guess. It is forecasting and measurement that considers the physical nature.

\section{The Susceptible Size of Coronavirus Spread}

In developing theories of the distribution of galaxies in the stellar system or of the distribution of centres of populations (animals, epidemics, and so on), it is convenient to regard the centres of the galaxies or the populations as points distributed in space. Consider an array of points distributed in a space $S$, where $S$ is a Euclidean space of dimension; $d \geq 1$. For each region $R$ in $S$, let $N R$ denote the number of points (finite or infinite) contained in the region $R$. The array of points is said to be distributed according to a stochastic mechanism if for every (measurable) region $R$ in $S$, $N R$ is a random variable. Thus, the array of points is said to be of Poisson type with density $v$ or to be distributed in accordance with a Poisson process with density $v$ if the following assumption is fulfilled: the numbers of points in nonoverlapping regions is an independent random variables. More precisely, for any integer $\mathrm{n}$ and $\mathrm{n}$ non-overlapping (disjoint) regions , the random variables are independent. Also, for any region $\mathrm{R}$ of finite volume, NR is Poisson 
distributed with mean, , where denotes the (d-dimensional Euclidean) volume of the region $\mathrm{R}$. The distributions in space of animals and plants are described by Poisson processes and generalized Poisson processes. In regards to the distribution of the nearest neighbour in a Poisson distribution of particles in space if particles are distributed on a plane in accordance with a Poisson process at a mean rate $v$ per unit area, and $T$ is the distance between an individual particle and its nearest neighbour, then $\pi v T 2$ is exponentially distributed with mean 1 . If particles are distributed in a three-dimensional space in accordance with a Poisson process at a mean rate $v$ per unit volume, and if $T$ is the distance between an individual particle and its nearest neighbor, then $4 / 3 \pi v T^{3}$ is exponentially distributed with mean 1 . Consequently, for $t>$ $0, f_{T}(t)=4 \pi v t^{2} e^{-\frac{4}{3} \pi v t^{3}}$ (Parzen Emanuel, 1967).

\section{What are the Forms of Causality?}

Is one variable sufficient to cause a phenomenon or is there a necessity for more than one variable to cause it? Is the difference clear enough between what might be called a phenomenon and what might be called a causative variable?

The result of throwing a coin may be called a single phenomenon in two forms. Also, the head may be variable, and the tail may be phenomena or vice versa. Consequently, physical phenomena may represent the beginning of a state of demise because they have lost an important part of their components. In addition, rare physical phenomena may need a relatively long period of time in order to repeat.

A linear variable $L$ cause a linear effect and linear variables $L$.

A linear variable $L$ cause a random effect and random variables $R$.

A random variable $R$ cause a linear effect and linear variables $L$.

A random variable $R$ cause a random effect and random variables $R$.

If this is true, they help to determine which one is a variable and which one is random and why?

If there were 100 infected people, 96 of them made contact with an infected person, 3 of them made contact with an unknown person, and 1 of them was without any contact. Therefore, the causes of infections could be:

1. Contact with unknown

2. Contact with an infected person

3. Dealing with the infectious non-human source (work and lifestyle) However, if there are different sources of infection and their transmission, it requires different methods to eliminate its seriousness. 


\section{Before and after the epidemic was discovered, are all populations susceptible to infection? Does the population in the same country have the same density?}

The total population is not used as one variable. This is because all its components do not have the same properties. There is a difference in density and a difference in lifestyle.

\section{How can primary infected people $I$ be defined?}

Infected people $I$ can be defined as the first people who were infected or people who were infected within the first 21 days in a country. The variables input for the mathematical model that measures the epidemic spread will be: population $N$, infected people $I$, susceptible people $S$, and the constant rate of infection. These variables are related to each other, either directly, inversely or randomly. Also, each variable has a definition or description.

If all the events are independent and have the same probability $P$ in any given day, then by the binomial theorem, the formula for the mean number of who will be infected is $\mu=\frac{r I S}{I+S}$.

To get a model of how the epidemic develops over time, the formula $\mu=r I S /(I$ $+S)$ is derived. Where the daily infective rate is $r I_{(t)} S_{(t)} / N$ and $N=I_{(t)}+S_{(t)}$. Thus, if $N$ is constant, $r / N=\beta$. Then,

$$
\begin{array}{ll}
\frac{d I(t)}{d t}=\beta I_{(t)} S_{(t)}=\beta I_{(t)}\left[N-I_{(t)}\right], & \text { equation } 1 . \\
w(t)=\frac{d I}{d t}=\frac{\beta N^{2} I_{(0)} e^{\beta N t}\left[N-I_{(0)}\right]}{\left[N-I_{(0)}+I_{(0)} e^{\beta N t}\right]^{2}}, & \text { equation } 2 .
\end{array}
$$

Furthermore, the epidemic curve peak $w\left(t_{\max }\right)=\beta N^{2} / 4$ if it was positive.

$w_{(t)}$ is considered a good measure of the strain placed on healthcare resources at time $t$. This is because it measures new cases that show up at the hospital. Therefore, $w_{(t)}$ is just another notation for $d I / d t$ (Meyer Walter, 1984).

In this model, the size of $N$ and $S$ was not calculated according to a specific formula. Also, the rate $r / N=\beta$ may be fixed everywhere in the world and may be variable depending on the nature of life, social relations, and technological development.

The number of connections that may cause infection depends on the nature of the work of the first people who were infected such as a restaurant waiter or doctor or any person who deals with the public significantly, which may exceed 100 connections a day. Since the virus is new and connections are open, the number of infected people depends on the connection with the active centre of the virus such as the food market or any centre that most people usually visit. The size of the $N$ is determined to start with the number of the initial infected persons in the possible connections at $t$. Hence, the community expands due to the outbreak and then shrinks due to preventive measures (Figure 1). 


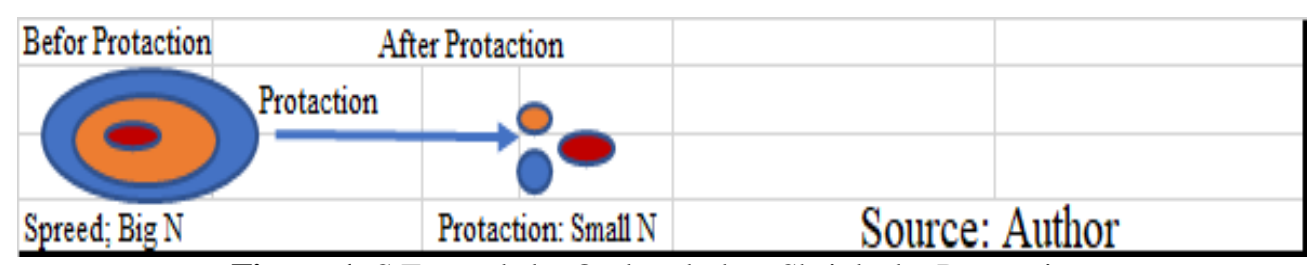

Figure 1. S Expands by Outbreak then Shrinks by Preventive.

The concept of homogeneity carries two meanings: biological meaning that accepts infection transmission such as homogeneity that accepts a blood transfusion and mathematical meaning since all units are equal in their susceptibility to be counted. At the same time, it is not homogeneous when one unit is able to remove another unit of the count. In the case of a population census of a city, the population is calculated at a specific time. This occurs when the city is closed at that time, and the entire population is homogeneous mathematically. However, in the case of an epidemic, the population is not homogeneous mathematically because increasing community members by an infectious person are completely different from increasing it by a noninfectious person. Therefore, there is a process of isolation for infected people.

The community in an epidemic case involves all individuals who have a probability of more than zero $(P>0)$ dealing with an infectious person. If all community has been infected, then the probability is $P=1$. This means that every individual connection were infectious. When the world's population is more than 7 billion people, the global community is supposed to be infinite. Therefore, it will be an open community until it begins to deal with infections. Not all community is susceptible to infection because the first infected person is an early warning that in turn leads the community to take preventive measures. For example, in the case of radioactive leakage, all community members within the circle of leakage will be affected by radiation.

From the definition of Poisson processes in space, $S$ is the susceptible people in the area of the epidemic circle and the epidemic is the ray. The lower the primary infection, the greater the number of members of the community that can be infected. For example, if there is a community of 10 people and one of them is infected $I_{(0)}=1$, the number of those susceptible to infection will be 9. This is greater than $I_{(0)}=5$, where the number of people who will be susceptible to infection are $10-5=5$. It is natural that the number of the first cases of epidemic infection is more than 1 . Within the incubation period and the diagnosis period of the disease, the number is greater. In addition, if $I_{(0)}$ was greater, then the probability of connection between an infectious person and another infectious person is more. If $I(0)$ was lower, then the probability of connection between an infectious person and a susceptible person is greater. Also, the spread of the virus depends on whether or not there is an active center. For $n$ communities of more than 2 people, there is a contact with this 
spread active center. The virus spread active center is a community of unlimited and variable random components. For this reason, $I_{(0)}$ is a small and almost constant value in the active center community while $S_{(0)}$ is a variable. Assuming the population is represented by small circuits with a potential density of 1 to 1000 people and if a specific country with a population of $60,000,000$ is divided into 1,000 societies, then the number of those societies is 60,000 . Also, the number of infections in each of them ranges from 0 (free from the epidemic) to 1,000 (everyone is infected).

If $I_{(0)}=10$, and the community of the virus spread active center $C$ was 100 daily, then $S 1_{(t)}$ are the 90 daily people who susceptible in the population by this center. If the community of the center was 1000 daily, then $S 2_{(t)}$ are 990 daily people who are susceptible in the population at $t$. In addition, if $S 1_{(t)}$ or $S 2_{(t)}$ is working in other centers, then the spread will be greater due to new spread centers. When a simulation program for the spread is in place, the spread is not the number of possible connections in all population, but rather within a circle $C$ of varying sizes $Z$ at $t$ (Figure 2).

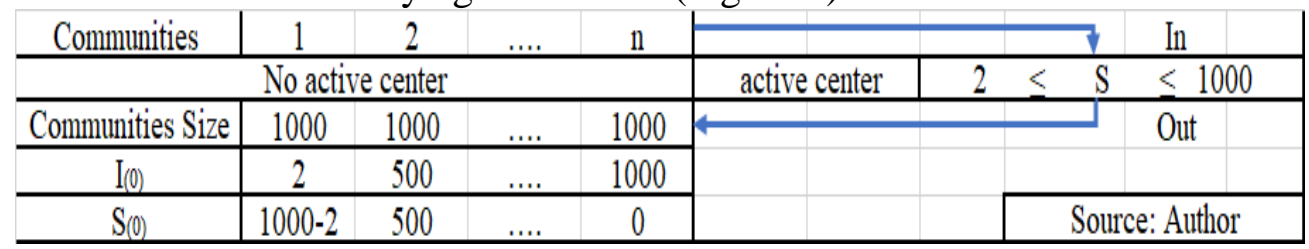

Figure 2. The Effect of $\mathrm{I}_{(0)}$ Size in the Size of $\mathrm{S}_{(0)}$ and the Active Center

To determine $N$ size in the epidemic model, first let $R$ be the ratio of infection to the total population.

$R=I_{(0)} / U$, where $U$ is the population.

After then, evaluate the parameter $\lambda$, and let the population be circuits that can be infected in the pre-isolation period. Therefore, the number of susceptible is $O$ if the pre-isolation period is 0 .

Suppose that the possibility of infection from an infected person to an uninfected person in a single day is $P=1 / 100$ for every day and suppose that the average number of infection connections is $X=1$ per day and the preisolation period $t=21$, then:

$\lambda=21 * 1 *(1 / 100)=0.21$

if $I=N$, then $P=0$ and $\lambda=0$ (Table 1).

Table 1. Parameter $\lambda$ Evaluated (by 21/100) as the Maximum Pre-Isolation Period

\begin{tabular}{c|c|c|c|c|c|c|c|} 
Pre-Isolation Period $\mathrm{t}$ & 0 & 1 & 2 & $\ldots$ & 14 & $\ldots$ & 21 \\
\hline (possible connections) $\mathrm{x}$ & $\mathrm{x} 1$ & $\mathrm{x} 2$ & $\mathrm{x} 3$ & $\ldots$ & $\mathrm{x} 4$ & $\ldots$ & $\mathrm{x}_{5}$ \\
\hline (probability of infection) $\mathrm{P}$ & $\mathrm{p} 1$ & $\mathrm{p}_{2}$ & $\mathrm{p}_{3}$ & $\ldots$ & $\mathrm{p}_{4}$ & $\ldots$ & $\mathrm{p}_{5}$ \\
\hline $\mathrm{t} * \mathrm{X} * \mathrm{P}$ & 0 & $X_{3} P_{1}$ & $2 X_{3} P_{2}$ & $\ldots$ & $14 X_{4} P_{4}$ & $\ldots$ & $21 X_{5} P_{5}$ \\
\hline
\end{tabular}

Source: Author.

For every $I_{(0)}$ in $\mathrm{U}$, there is a value for $S_{(0)}$ in $R$. 
Let $I_{(0)}=x$, and $R=f(x)$, then $(g \circ f)(x)=g(f(x))=S_{(0)}$.

$S_{(0)}=\left\{\begin{array}{rr}S_{(0)}, & S_{(0)}>\lambda \\ 0, & S_{(0)}=\lambda\end{array}\right.$

$S_{(0)}=\lambda / R$, because $R \neq 0$ so, $S_{(0)}=0 . /(R+\gamma)$, where $\gamma=1 / U$

If the pre-isolation period is 21 days, then $S_{(0)}=0.21 /\left(I_{(0)} / U\right)$. Here the value of $\gamma$ can be neglected. If $U=I$, then:

$R=1$ and $\mathrm{S}=\lambda=0$. So,

$N=S_{(0)}+I_{(0)}$ and, if $\lambda=0$ then,

$S=0$ and $N=I$ (Table 2).

Table 2. Using the parameter $\lambda$ to evaluate $S$ size

\begin{tabular}{c|c|c|c|c|c|c|c|} 
Area & Population Size Under Epidemic U & $\mathbf{I}(\mathbf{0})$ & $\mathbf{R}=\mathbf{I}(\mathbf{0}) / \mathbf{U}$ & Parameter $\boldsymbol{\lambda}: \mathbf{0} \leq \boldsymbol{\lambda}<\mathbf{1}$ & $\mathbf{S}_{(\mathbf{0})}=\boldsymbol{\lambda} /(\mathbf{I}(\mathbf{0}) / \mathbf{U})$ & $\mathbf{N}=\mathbf{S}(\mathbf{0})+\mathbf{I}(\mathbf{0})$ \\
\hline & & $74,000,000$ & 2 & 0.0000000270270 & 0.21 & $7,770,000$ & $7,770,002$ \\
Germany & & $74,000,000$ & 50 & 0.0000006756757 & 0.21 & 310,800 & 310,850 \\
Area: Urban & \multirow{2}{*}{$74,000,000$} & $74,000,000$ & 60 & 0.0000008108108 & 0.21 & 259,000 & 259,060 \\
+(1/2) Non & & $74,000,000$ & 84 & 0.0000011351351 & 0.21 & 185,000 & 185,084 \\
Urban & & $74,000,000$ & 1,000 & 0.0000135135135 & 0.21 & 15,540 & 16,540 \\
\hline
\end{tabular}

Source: Author.

Using equation 2, and assuming that at $t=0$ the coronavirus outbreak in Germany $I_{(0)} \leq 83$ (it can be 2 to 1000 ), $r=0.15$ for the population of $74,000,000$. This paper evaluates $U$ from the total population of all areas that have a spread active center or a daily connection with it. Thus, it is similar to those who live in a city and work in the active center or who work in these cities simultaneously. The true population of Germany is more than eighty three million $(83,000,000)$, but this paper will assume that the population is the urban population plus half of the non-urban population as a study area. Thus, the epidemic cases spread to about seventy four million people (74, 000,000). In Hubei case, this paper assumed $r=0.15$, and $I_{(0)} \leq 150$ for the population of 58,600,000 (Figure 3).

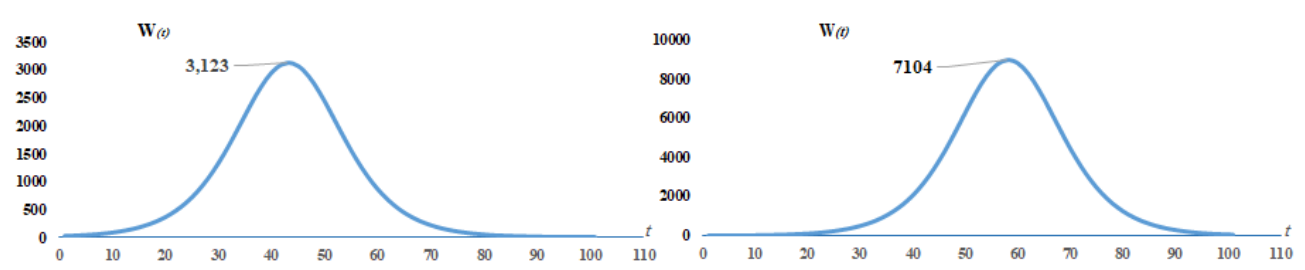

Figure 3. Hubei $\mathrm{w}_{(\mathrm{t})}$ Curve (left) and Germany $\mathrm{w}_{(\mathrm{t})}$ Curve (right). Source: Author.

Consequently, the highest point in the $\mathrm{w}(\mathrm{t})$ curve or $w\left(\mathrm{t}_{\max }\right)$ gives the largest number of infections per day. $w_{(t)}$ curve shows the expected number of infected cases, while the hospital shows the discovered cases. Therefore, the faster the discovery, the higher the numbers (Figure 4). 


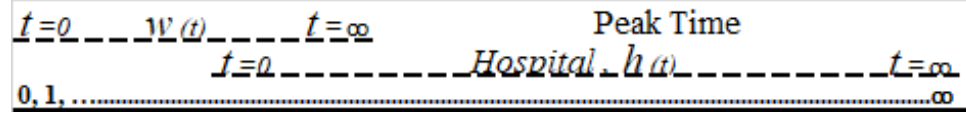
Source.Author

Figure 4. The Distribution of the Number of Discovered Cases by Days is Different from the Distribution according to the $w_{(t)}$ Curve.

However, the important point here is that hospitals may receive greater or lesser than this number in one day. This is because they may receive a total of several cases in one day. This is in addition to the variation in the length of period before the symptoms of the disease appear. The number of announced daily new cases is affected by the maximum capacity of hospitals, as well as the method for calculating cases that may depend on the total number of those who were admitted to the hospital and those who left the hospital in addition to the diagnosis method. Therefore, the numbers generated by the model may differ slightly from those declared officially. The curve of total cases recorded in Figure 5 (left) shows a deviation on February 12. This is seen with a jump in the number of cases recorded and is the result of a change in the method of diagnosis, which constitutes a statistical defect. This is because the new cases recorded on February 12 should be distributed over the previous days. Also, when the epidemic period is divided into 4 days to 7 days, it gives manycurves as seen in Figure 5 (right). At the first downward curve, there is a good indication that the epidemic is beginning to decrease.
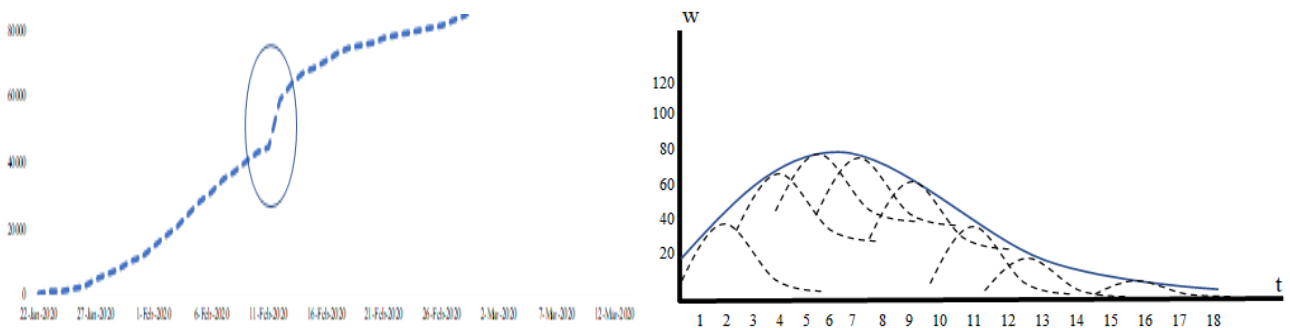

Figure 5. Total Cases of Coronavirus Curve from 22-Jan-2020 to 13-Mar-2020.

Worldometer (Left). Upward Curves at the Beginning of the Epidemic and Downward Curves at the End of the Epidemic (Right). Source: Author

\section{The Age of the Epidemic and Reliability System}

To measure the epidemic age, time, $t \geq 0$ and $n$ is the duration of incubation of the virus (Figure 6).

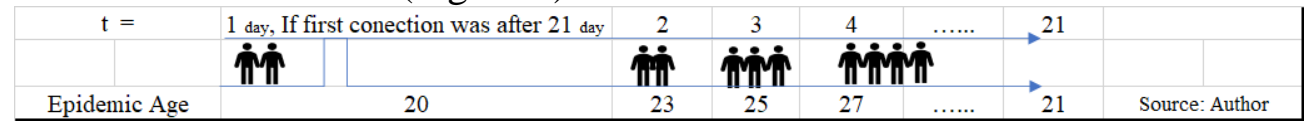

Figure 6. Total Epidemic Age

The incubation periods of the epidemic plus the period required to discover the disease until the patient is isolated is 21 days. Also, the number of affected people at $t$ has no effect in the age of the epidemic at $t$. If the community consist of 1000 people and all of them are infected at the same 
time, then $I_{t}=1$. Also, if $n_{t}=0$, infected at $\mathrm{t}$ is equal to 0 . Hence, the age of the epidemic $G$ can be measured by the total of these periods for every new infected person as follows:

If the epidemic age $n_{\mathrm{t}}$ at $t=1$ which is the total periods $+\Delta \mathrm{n}$, then:

$$
\begin{aligned}
& n_{\mathrm{t}}=n_{\mathrm{t}}+\Delta \mathrm{n} \text { and } \Delta \mathrm{n}=n_{\mathrm{t}}-n_{t-1} . \\
& n_{1}=n_{1}+\Delta \mathrm{n}=n_{1}+\left(n_{1}-n_{0}\right) . \\
& n_{2}=n_{2}+\Delta \mathrm{n}=n_{2}+\left(n_{2}-n_{1}\right)+\left(n_{2}-n_{0}\right) . \\
& n_{m}=n_{m}+\Delta \mathrm{n} \text { and } \Delta \mathrm{n}=\sum_{t=1}^{m}\left(n_{m}-n_{m-t}\right) \\
& G=n_{m}+\sum_{t=1}^{m}\left(n_{m}-n_{m-t}\right) . \text { Where is } 0 \leq n_{i} \leq 21 \text { (Figure 7). }
\end{aligned}
$$

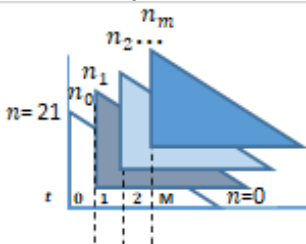

Figure 7. The Addition of Every Infected Default Age Starts from 21 Days

Also, the addition of life lengths or convolution can be found using the statistical theory of reliability and life testing by assumed independent units ages $x_{1}, x_{2}, \ldots, x_{n}$ with $f_{1}, f_{2}, \ldots, f_{n}$ distributions, where $F$ is distribution function of $x_{1}+x_{2}+\cdots+x_{n}$ and $\int_{0}^{t} F_{2}(t-x) d F_{1}(x)$, this means that if the first unit fails at $x$ time earlier than the moment $t$, while the second unit is succeeding during the next period $t-x$, then the sum of the two ages does not exceed $t$, and $F$ is the distributions convolution $F=F_{1} * F_{2}$ (Barlow \& Proschan, 1975). The epidemic performs natural replacement when the infection is transmitted from one person to another, which gives it a longer life but more $S$ size (Figure 8 ).

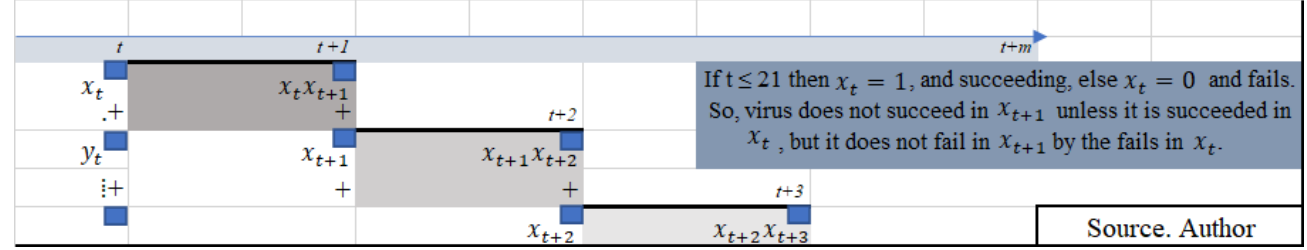

Figure 8. Virus Reliability System is Parallel Series Parallel System.

The reliability system of the epidemic $\varphi$ is:

$\varphi=x_{t} \cap x_{t+1} \cup x_{t+1} \cap x_{t+2} \cup x_{t+2} \ldots+y_{t} \cap y_{t+1} \cup y_{t+1} \ldots$

$\varphi=x_{t} \cdot x_{t+1}+x_{t+1} \cdot x_{t+2}+x_{t+2} \ldots+y_{t} \cdot y_{t+1}+y_{t+1} \cdots$

\section{Optimal Solution of Two-Person Zero-Sum Games and Decision Trees}

The dependence on medication differs from the dependence on prevention or vaccine because the medicine comes after the disease. This 
involves more costs and sick leave. Since the epidemic affects the human respiratory system, the protection from the epidemic includes protecting the respiratory system from allergies. Allergies related to the ear, nose, and throat, in particular, affects the ability of breath. Breathing is also associated with will and confidence. Since the allergy precedes inflammation and common cold, it may be considered as one of its causes. Thus, protection is a strategic step against the epidemic. Immune boosters and antibiotics which serve as antiallergy also play a significant role. Also, one of the most important strategic steps is to prevent the epidemic from spreading in traditional hospitals because the risk of the epidemic will be great especially for patients. More so, doctors may catch an infection and transfer it to other patients. However, this can be prevented by building temporary hospitals for the epidemic.

The game theory in the problem of epidemic is a game that only has one player as the human, while the other player is termed as nature. However, this paper does not encourage this designation because humans should always reconcile with nature. In addition, this paper does not like to express that epidemic plays the role of natural selection because the moral and mental criterion is above the physical criterion. The epidemic from its birth is spread through humans as the number of people affected by the epidemic is an enumeration for it. At the same time, every life case of the epidemic is matched by one illness or death for humans. Thus, humans seeks to end the epidemic using supportive therapy $M$, vaccine $V$ and disinfection, and protection $D$.

Therefore, let $M$ be the recovery rate. This paper found an excel program to calculate the recovery rate by assuming that the number of new cases is a random variable. Hence, daily reading of infected, recovered, and death cases are used as a baseline number that is updated based on the reported data. The number of infection cases the next day is:

$y_{t+1}=\left(x_{t}-q_{t}-d_{t}\right)+\left(\left(x_{t}-q_{t}-d_{t}\right) \frac{n_{t}}{x_{t}}\right)+\left(\frac{n_{t}}{x_{t}} \mathcal{L}\right)$, while the number of recovery cases the next day is: $q_{t+1}=\left(q_{t} / x_{t}\right) x_{t+1}$. Then this is applied to the remaining days until $y=0$. Where $x$ : total infection cases today, $q$ : total recovery case today, $d$ : total death case today, $n_{t}$ : new cases today, and $\mathcal{L}$ : the change in the rate of new cases. If all patients take the same medical care, then the recovery rate is $M=0.94$. Table 3 shows the program with input and output. 


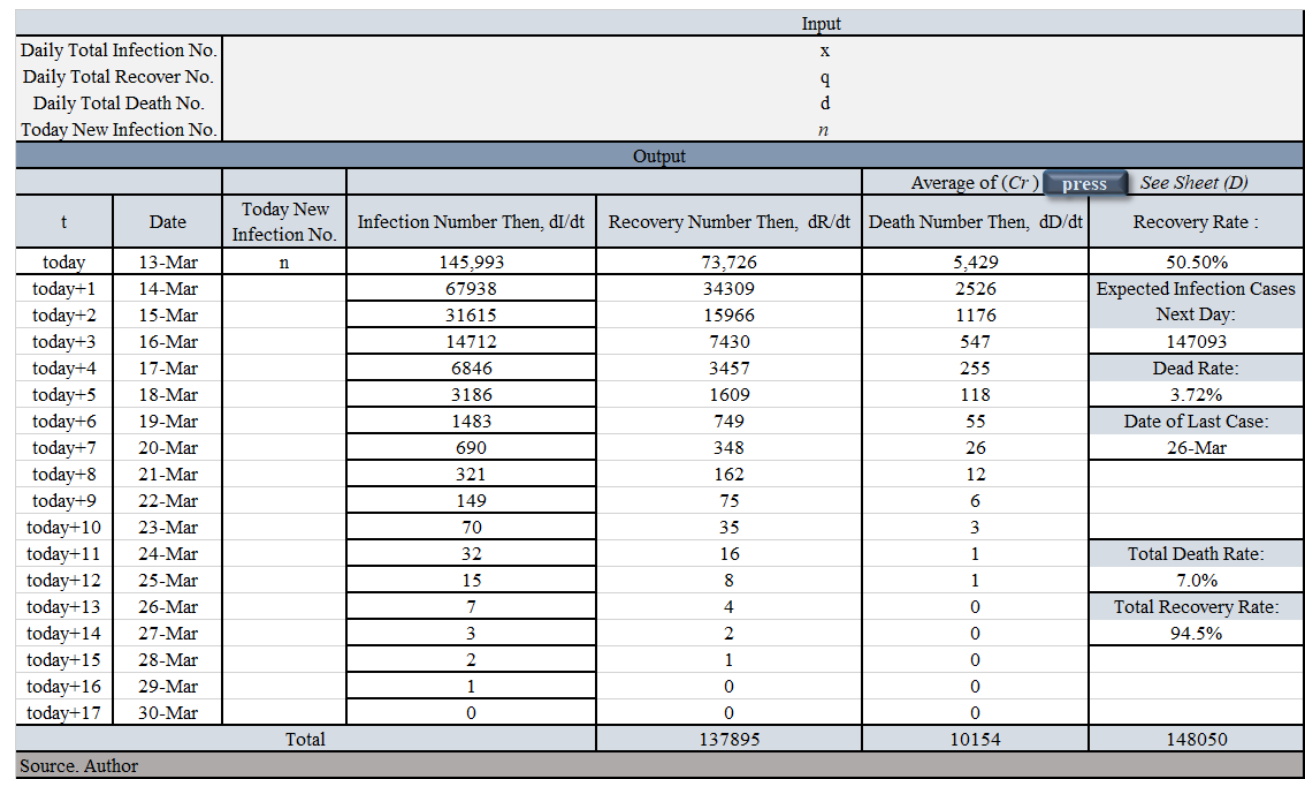

Table 1. Computer Program to Calculate the Recovery Rate

Assuming that the game against the epidemic will be in the following situations: before attacking the body, when attacking the body, and after attacking the body and if the effect of $M=0.94, D=0.99, V=1$, then the results that may arise by this are recovery or death, protection or infection, and vaccinated or not. The epidemic naturally takes behavior to increase its default age. At the same time, humans are supposed to take a defensive stand in the face of the pandemic. Therefore, the epidemic seeks to gain, while humans seek to avoid or reduce losses. Subsequently, the payoff matrix will be:

$$
\begin{aligned}
& B \\
& A\left|\begin{array}{c|cccc}
x_{1} & y_{2} & \ldots & y_{n} \\
x_{2} & a_{11} & a_{12} & \ldots & a_{1 n} \\
x_{21} & a_{22} & \ldots & a_{2 n} \\
\vdots & \vdots & \vdots & & \vdots \\
x_{m} & a_{m 1} & a_{m 2} & \ldots & a_{m n}
\end{array}\right| \\
& \text { Human }
\end{aligned}
$$

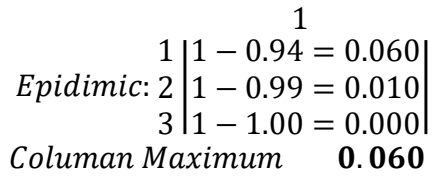

$$
\begin{aligned}
& \text { Row Minimum } \\
& \begin{array}{r}
\mathbf{0 . 0 6 0} \text { Maximin } \\
0.010 \\
0.000
\end{array}
\end{aligned}
$$

Minimax

The saddle point here is 0.060 and the maximin $=\operatorname{minimax}=0.060$. Here, humans should choose this strategy against the epidemic even if they had a vaccine because this choice represents the critical line of defense. Also, if they had a vaccine and an accurate medicine, they should choose the protection. In addition, $M, V$ and $D$ may take many values when considering many states with deferent situations such as the level of supportive therapy and the level of protection. Hence, "if there is no saddle point, the failure of 
the minimax-maximin (pure) strategies, in general, which gives an optimal solution to the game leads to the idea of using mixed strategies. In this case, humans play all their strategies according to the predetermined set of probability. Let $x_{1}, x_{2}, \ldots, x_{m}$ and $y_{1}, y_{2}, \ldots, y_{n}$, be the row and column probabilities of $A$ and $B$, respectively. Then, $\sum_{i=1}^{m} x_{i}=\sum_{j=1}^{n} y_{j}=1, x_{i}, y_{j} \geq 0$ for all $i$ and $j . "$ (Hamdy a. Taha., 1989).

A graphical representation of the decision problem can be made by using a decision tree. The decision tree in the level of health is tree I (Figure 9).

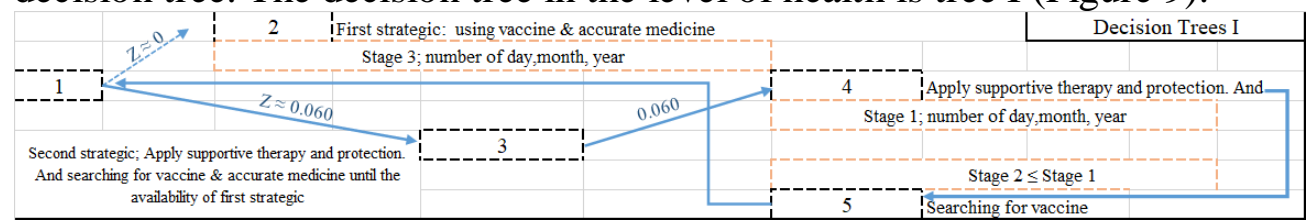

Figure 9. Decision Tree in the Level of Health, Bold Line

The decision taken is to use the second strategy that includes searching for vaccine $\&$ accurate medicine until the first strategy is available which involves using the vaccine \& accurate medicine. The second strategy has two parts: searching for vaccine and applying for supportive therapy and protection. Since time is a determining element, the period between when a virus begins to spreads and when a vaccine is available must include decisions that reduce the chances of spreading and infection.

The decision tree in the level of economy is tree II (Figure 10).

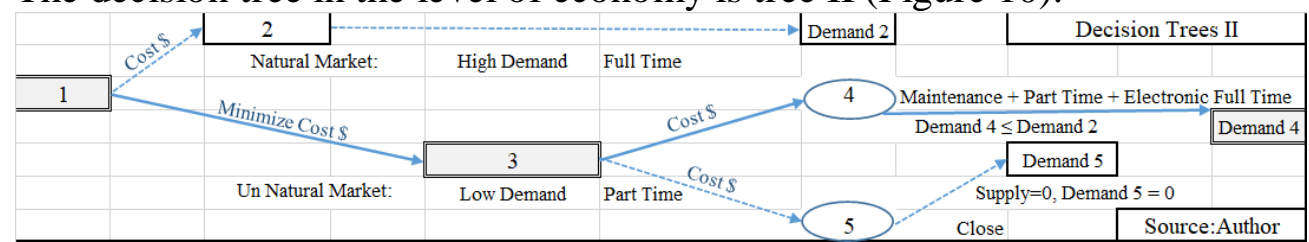

Figure 10. Decision Tree in the Level of Economy, Hold Line

Furthermore, suppose the demand x per period on a certain commodity is given by the continuous probability density function $f(x)$, if the amount stocked at the beginning of the period is not sufficient, shortage may occur. If too much is stocked, extra inventory will be held at the end of the period. This means that both situations are costly. The first reflects loss of potential profit and loss of customers' goodwill, while the second reflects an increase in the cost of storing and maintaining the inventory. To balance these two conflicting cost, it is important to determine the level of stock such that the expected shortage quantity does not exceed $A_{1}$ units and the expected excess quantity does not exceed $A_{2}$ units. To express this mathematically, let $l$ be the stock level to be determined and the expected shortage quantity $=\int_{l}^{\infty}(x-$ 
l) $f(x) d x \leq A_{1} \quad$ while $\quad$ the expected excess quantity $=\int_{0}^{l}(l-$ $x) f(x) d x \leq A_{2}$. (Hamdy Taha, 1989).

In addition, as humans always sought to choose the shortest path, the epidemic also takes advantage of this to spread quickly. Therefore, in the case of an epidemic, the shortest path has some conditions. To reduce the chances of spreading the disease, selecting direct paths that avoid bifurcations and traffic with several points is needed. Although this may reduce profits and increase the cost of transportation projects, this also reduces the spread of the disease period. Therefore, the shortest path should use the strategy of the direct and returns path.

Assuming city represents a node $i$, and passenger $p, p \in i$ for $i=1, \ldots, n$. And $i$ $=2, \ldots, n-1$, for every $p$ stay period $\omega$ is $14 \leq \omega \leq 21$ day (Figure 11).

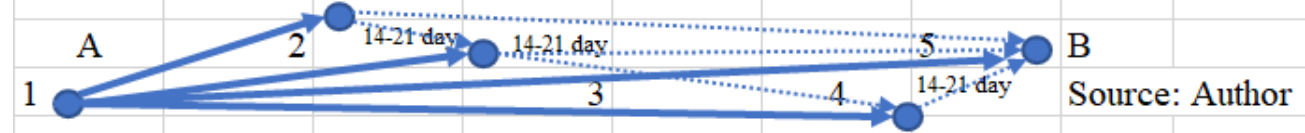

Figure 11. The Trip is Only for Citizens of the City A or B, Bold Line

Furthermore, for schools or any manner of work such as schools, work should be a day for school $A$ and a day for school $B$. Every school should also be divided into groups, a day for group $A$ and a day for group $B$. A full day or 12 hours should be used to reduce the density. If the size of work is $K$ and the size of worker is $W$, then the total job for everyday is $J=K * W$. On the other hand, If $J=1 / 2 W$ and the online work is $1 / 2 J$, then $J$ in epidemic case + online work $=J$ in natural case. This should include the reallocated work (Figure 12).

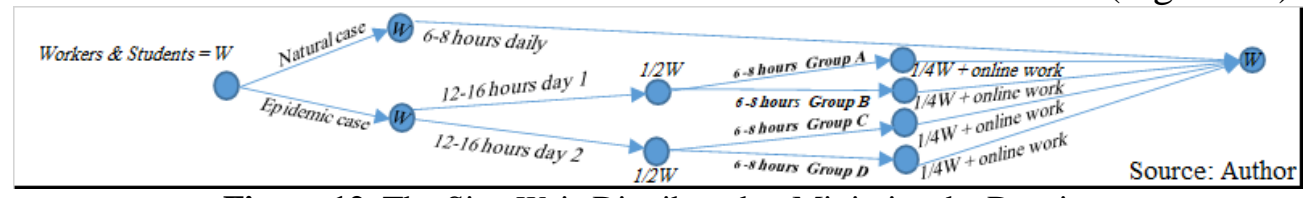

Figure 12. The Size $W$, is Distributed to Minimize the Density

If the outbreak is followed by a downturn in the economy, this comes to an end once the epidemic stops. The recovery is accompanied by an additional driving value that was not achieved during the downturn such as the state of fasting and then breaking the fast. This value can be deduced from the following equation: GDP at the beginning of epidemic - GDP at the end of the epidemic. This happens if there is a stimulus policy or if the economic system is protected from any widespread effects of the epidemic for natural resources and human resources, as well as any important values and if bankruptcies do not occur on a tangible scale. Consumer behavior in a pandemic is likened to the behavior in a case of war. On the other hand, the behavior of producers is directed to the necessities that the consumer cannot do without such as medical products. The state, in addition to providing necessities, should meet the needs 
of the consumers during the outbreak of the epidemic. Although the world is trying to overcome the hurricanes threats because of its economical harms, the economic interaction with the virus should not create a state of panic. In general, it should control the negative effects of biotechnology that tamper with the environmental balance which released genetically modified microorganisms that resulted from the development of organisms to survive in difficult natural environments (Jean-jacques Salmon, Francisco Sagasti \& Céline Sachs -Jeanet, 1998).

\section{Conclusion}

For good models, this paper defines the population in an epidemic case as a circle of $2-1000$ person and $2 \leq I_{(0)} \leq 1000$. For $n$ communities, there is a contact with the spread active center. The virus spread active center is a community of unlimited and variable random population. As a result, $I_{(0)}$ is small and almost constant. Aside that, there is no virus active center or the cases are miscellaneous. This paper found a new parameter $\lambda$ to evaluate the size of $S$ used in the epidemic model. The primary infectious I cannot be less than two people because one person may have absolutely no contact. Also, this paper does not consider $N$ as all populations but instead considers $N$ as an area. Thus, this paper recommends closing any active center as soon as possible and to prevent any active center to be initiated.

This paper found an equation that measure the age of the epidemic as: $G=n_{m}+\sum_{t=1}^{m}\left(n_{m}-n_{m-t}\right)$. Therefore, the longer the epidemic age, the greater the size of the susceptible people $S$. Also, this paper found that the virus reliability system is a parallel series parallel system where the virus does not succeed in $x_{t+1}$ unless it succeeds in $x_{t}$. However, it does not fail in $x_{t+1}$ if it fails in $x_{1}$. Thus, this is in addition to evaluating $r$. This paper concluded that the hospitals may receive greater or less than the highest point in the $w_{(t)}$ curve. Subsequently, the study recommends that the speed of discovering disease should be greater than the speed of disease spread. In this equation; $y_{t+1}=\left(x_{t}-q_{t}-d_{t}\right)+\left(\left(x_{t}-q_{t}-d_{t}\right) \frac{n_{t}}{x_{t}}\right)+\left(\frac{n_{t}}{x_{t}} \mathcal{L}\right), q_{t+1}=\left(q_{t} / x_{t}\right) x_{t+1}$.

$x_{t+1}$, is used to calculate the recovery rate in the game model that suggest the optimal solution for the game by 0.060. It is also seen as a strategic choice for humans and the epidemic. It was concluded that the decision trees should be taken into consideration as well as the second strategy which includes searching for vaccine \& accurate medicine. It also involves the application of supportive therapy and protection. Since time is a determining factor, a decision should be taken to reduce the spread of the virus between the periods beginning from the spread of the virus to the availability of a vaccine. Therefore, the following precautions should be strictly adhered to: 
- Work to sterilize or disinfect all possible places that may help the virus to spread. Also, hospital buildings should be isolated from traditional hospitals to avoid the spread of the virus. This should be done with the aim to keep traditional hospitals away from infection. In addition, an anti-allergy should be used as it helps to avoid the increased chance of infection due to sneezing resulting from cold infections. This occurs mostly in food markets and in warm areas where the markets are air-conditioned, where open refrigerators may cause a cold shock. Also, people are advised to take immune-boosting foods because this helps protect people even more if they were carriers of the virus before it was discovered. Also, masks which are similar to tissues should be used only once and then disposed of, so as not to be the cause of infection because it absorbs the spray.

- Testing of anyone arriving and departing a country instead of closing the borders. Thus, for airlines, this paper recommends selecting direct paths that avoid bifurcations and traffic. It is also not true to limit roaming to a limited period, as this may increase congestion, which in turn may increase the spread of infection, especially in shopping centres. An effective approach to curb the spread of this virus is to avoid overcrowding and observing social distancing.

- Decisions should be taken that stimulate the confidence to confront the virus, protect the economic system from any widespread effects of the epidemic for natural resources, and prevent any bankruptcies to occur on a tangible scale. In addition, when the financial markets fall sharply, companies should rebuy their shares.

- Decisions that focuses on reducing costs and choosing partial routine work and full electronic work should be adopted. Reallocation the work, this transfer the cost of work-related to stores that do not work fully to another activity within the activities of the company that works seasonally, and workers should be subjected to virus testing. Likewise, marketing activity during the epidemic should be done in a way that has a positive impact on customers. At the end of the epidemic, a stronger business model should emerge that should attract more customers.

\section{References:}

1. Abdul Sattar Al Alusi (2003). Operational Research Techniques: Quantitative Methods for Decision Making (1st ED). UAE: ITTIHAD University. Units (8).

2. Alain Anderton (1995). Economics (2nd ED). Oxford: Causeway Press Limited. Units (106). 
3. Alan Garfinkel., Jane Shevtsov \& Yina Guo (2017). Modeling Life: The Mathematics of Biological Systems (1st ED). Springer International Publishing AG. Switzerland: Units (1,6,7).

4. Barnes, B. (2015). Mathematical Modelling with Case Studies (3ed ED). CRC Press. Kindle Edition. Units (5).

5. Barlow, R.E. \& Proschan, F. (1975). Statistical Theory of Reliability and Life Testing (2nd ED). USA: Holt, Rinehart, and Winston. Unit $(4,6,8)$

6. Bender, E.A. (2000). An Introduction to Mathematical Modeling(1st ED). Dover Publications, INC. New York. Units $(1,2)$.

7. Brauer, F. (2010). General compartmental epidemic models. Chin. Ann. Math. Ser. B 31, 289-304. https://doi.org/10.1007/s11401-0090454-1

8. Chowell, G., Tariq, A. \& Hyman, J.M. A novel sub-epidemic modeling framework for short-term forecasting epidemic waves. BMC Med 17, 164 (2019). https://doi.org/10.1186/s12916-019-1406-6

9. Dominick Salvatore \& Eugene Diulio (2003). Principles of Ecomomics (1st ED). USA: McGraw-Hill Education. Units $(3,9)$.

10. Earl, W.S. (1988). Calculus with analytic geometry (2nd ED). USA: P W S. Kent Publishing Company. Units $(1,2,3,4,5,6,9,17)$

11. Eck, CHG. \& Peter, K. (2017). Mathematical Modeling (1st ED). Springer International Publishing AG. Switzerland: Unit $(1,4)$

12. Eric Rasmusen (1995). Games and Information (2nd ED). USA: Blackwell Publishers Inc. Unit (3).

13. Gu, J., Gao, Z. \& Li, W. (2011). Modeling of epidemic spreading with white Gaussian noise. Chin. Sci. Bull. 56, 3683-3688. https://doi.org/10.1007/s11434-011-4753-z

14. Hamed, M.A. (2020). An overview on COVID-19: reality and expectation. Bull Natl Res Cent 44, 86. https://doi.org/10.1186/s42269-020-00341-9

15. Hamdy, A.T. (1989). Operation Research (4th ED). New York: Macmillan Publishing Co., Inc. Units (11).

16. Howard Anton \& Bernard Kolman (1982). Mathematics with Aplication for The Management Life and Social Science (1st ED). USA: Anton Textbooks, Inc and KTI. Units $(1,2,4,810,11,12,13,14,15)$

17. Jean-jacques Salmon, FRS. \& Céline Sachs, J. (1998). The Uncertain Quest:science: Technology, and Development(1st ED). Japan: United Nations. Units (13).

18. Lahodny, G.E. \& Allen, L.J.S. (2013). Probability of a Disease Outbreak in Stochastic Multipatch Epidemic Models. Bull Math Biol 75, 1157-1180. https://doi.org/10.1007/s11538-013-9848-z 
19. Liu, W.J., Bi, Y., Wang, D. et al. (2018). On the Centenary of the Spanish Flu: Being Prepared for the Next Pandemic. Virol. Sin. 33, 463-466. https://doi.org/10.1007/s12250-018-0079-1

20. Meyer, WJ. (2004). Concepts of Mathematical Modeling (2nd ED). Dover Publications, INC. New York.. Units (5).

21. Parzen, E. (2015). Stochastic Processes (3ED ED) . Dover Publications, INC. New York. Units (1).

22. Paul Martin (1997). The Sickening Mind: Brain, Behaviour, Immunity and Disease (1st ED).UK: Harper Collins Publishers. Units $(2,4,5)$.

23. Ravindran, A.R. (2008). Operations Research and Management Science Handbook (1st ED). USA: CRC Press. Units $(4,5,6,17,20,22)$.

24. Sadig Majed Mohammad (1991). Operation Research (1st ED). University of BASRAH. Units (7).

25. Willimam \& Cochran (1977). Sampling Techniques (3RD ED ). USA: John Wiley \& sons, Inc. Units $(1,2,4,7,13)$.

26. Worldometero. https://www.worldometers.info/coronavirus/

27. Zaven, A.K. \& Edward, J.D. (1998). Modern Statistical, Systems, and GPSS Simulation (2nd ED). USA : CRC Press LLC. Units $(1,3,6,8)$. 